\title{
Compare combination allograft material to DFDBA in alveolar ridge preservation
}

\begin{abstract}
The aim of this split mouth clinical study was to compare DFDBA to combination allograft that is $70 \%$ FDBA and $30 \%$ DFDBA in alveolar ridge preservation. Changes in ridge dimension were evaluated, as was graft consolidation, ability to place implants, and additional grafting needs.

Methods: 20 extraction sockets in 6 patients who presented to the School of Dental Medicine were selected to be part of the study. These patients required 20 extractions with ridge preservation, with the eventual goal of being restored with dental implants. Extraction sockets were randomly assigned to either the control group (DFDBA) or test group (combination allograft). Immediately after extraction, a limited CBCT was taken to evaluate the socket. The extraction sockets received the assigned graft material, was covered with fast absorbing collagen dressing, and sutured with polyglactin. After 6 months, a second scan was taken. 3 -D rending software was used to compare the two scans and measured horizontal dimensional changes to the ridge.

Results: The changes were found as a loss of $0.67 \mathrm{~mm}$ for the test group and $0.74 \mathrm{~mm}$ for the control group. Graft integration was excellent for both the control and test groups. Implant placement was possible without any additional augmentation in these sites that went under ridge preservation

Conclusions: Extraction followed by alveolar ridge preservation using either demineralized freeze dried bone allograft or a 70:30 combination allograft resulted in clinically negligible changes in alveolar ridge dimension. There were minimal differences between the two grafting materials when measuring changes in alveolar ridge dimension using $\mathrm{CBCT}$.
\end{abstract}

Volume 9 Issue 5 - 2018

Famili P, Sanju P Jose

Department of Periodontics, University of Pittsburgh, Pittsburgh, Pennsylvania, USA

\begin{abstract}
Correspondence: Famili P, Department of Periodontics, University of Pittsburgh, Pittsburgh, Pennsylvania, USA, Email paf@pitt.edu
\end{abstract}

Received: July 31, 2018 | Published: October 16, 2018

\section{Background}

A systematic review on alveolar ridge preservation evaluated the effect of alveolar ridge preservation in comparison to non-grafted socket healing. ${ }^{1}$ In addition to looking at the changes in ridge dimension after extraction with ridge preservation or extraction without grafting; this group also looked to evaluate histological characteristics in both groups. 11 studies included histological analysis via biopsies of trephine cores taken out at implant placement. In these 11 studies, 149 sockets healed without any grafting, while 181 sockets were grafted at the time of extraction. ${ }^{2}$ studies reported significantly higher trabecular bone with ridge preservation when compared to unassisted socket healing. Significantly higher connective tissue in the non-grafted sites were reported by 2 studies. ${ }^{2}$ Significantly more vital bone in the nongrafted sites were reported by one study. ${ }^{3}$ Other histomorphometric parameters evaluated in these studies were not statistically significant. As is evident here, no particular advantage histologically, in terms of more vital bone, less connective tissue, or denser bone, has been demonstrated in various studies comparing grafted extraction sockets with non-grafted sites. ${ }^{3}$

The potential of using a combination allograft for socket preservation may allow the clinician to reap the benefits of both DFDBA and FDBA, leading to both volume stability and increased quality/composition of bone. No reported study has compared combination allograft to DFDBA in terms of ridge dimension and graft integrity. This pilot study will compare DFDBA to a novel combination allograft of FDBA/DFDBA in alveolar ridge preservation.

\section{Materials and methods}

In this randomized clinical study, we recruited, treated and followed patients presenting to the University Of Pittsburgh School Of Dental Medicine for extractions and implant placement. This study was conducted by the principal investigator at the University of Pittsburgh. The clinical study protocol and materials have been approved by the IRB (REN16100237/PRO14110066) of the institution. Informed consent was obtained.

Patients: In this pilot study with a split-mouth design, we recruited enough patients to have 10 control sites and 10 test sites. Patients that are under the medical care of a physician for any health condition which would contraindicate successful simple extraction will be excluded, including uncontrolled metabolic conditions, bleeding disorders, and smoking. All other subjects may be included. These patients were selected such that each patient would need at least two such extractions on bicuspid area, occlusal clearance and adjacent teeth were evaluated. Other inclusion criteria were: extraction socket was aligned with future implant placement, adequate occlusal space was available for future restoration, and bone loss was less than $50 \%$. Extraction sites were randomly assigned in each patient to either combination allograft or DFDBA immediately prior to the surgical procedure.

Surgical procedure: Patients were anesthetized with $2 \%$ lidocaine with 1:100,000 epinephrine for the mandible and 4\% septocaine with 1:100,000 epinephrine for the maxilla. Extractions were done in a 
minimally invasive manner, using luxators and elevators, without raising a mucoperiosteal flap in most situations. In cases where buccal dehiscence exists, mucoperiosteal flap was tunneled to place fast absorbing collagen dressing along this dehisced aspect to contain graft material. Sockets were curetted and irrigated adequately. Site-specific limited volume cone beam volumetric tomography scans were taken immediately after the extractions. The fresh extraction sockets received the assigned graft material, and were covered with fast absorbing collagen dressing. The oral tissues overlying the sites were closed and sutured with resorbable polyglactin sutures for secondary closure in a similar fashion. All patients were instructed in standard post-operative procedures.

Graft materials: A combination allograft was used in the test sites. This is a newly developed blend of $70 \%$ mineralized freeze-dried and $30 \%$ demineralized freeze-dried cortical bone. The particulate size range was $0.25-1 \mathrm{~mm}$. Demineralized freeze-dried cortical bone allograft was used in the control sites. The particulate size range was $0.25-1 \mathrm{~mm}$.

Follow-up examinations: Subject recall appointments for site evaluation were at one week, one month and sixmonths after extraction. Sutures were removed after one week, and post-operative instructions given again. A second site-specific limited volume cone beam volumetric tomography scan was taken after sixmonths of healing period.

CBCT evaluation and clinical examination: Baseline CBCT (Accuitom 3 D(R) Morita Kyoto, Japan) FOV (New Tom 3G (R) taken immediately after extraction were compared to the CBCT taken 6 months post-extraction, to assess changes in alveolar ridge dimensions, in terms of ridge width. The CBCT taken at 6 months post-extraction was also used to evaluate graft integration into the extraction sockets. Clinical exam was done at the time of implant placement to evaluate ridge integrity and quality of bone. The need for additional augmentation during implant surgery was also be evaluated.

\section{Results}

Total of 10 sets of extraction sockets in 6 patients were used in this split mouth study. Immediately following extractions, a site-specific limited volume cone beam volumetric tomography scan was taken for all 20 extraction sockets. These scans were then analyzed using 3-D rendering software, and measurements were made for width. In case of thin buccal plate or missing buccal plate, measurement was made from the most coronal aspect of the existing cortical buccal plate, appearing as a dense, thick radiolucent outline on the software rendering. All extraction sockets received the randomly assigned graft material. In the case of missing buccal plate, the flap was undermined, and fast absorbing collagen dressing was placed on the buccal aspect as well, to contain the defect. All patients healed uneventful without any infections or other issues. Patients were recalled at the appropriate intervals, and a second site-specific limited volume cone beam volumetric tomography scan was taken for all 20 extraction sockets. These scans were also analyzed using 3-D rendering software. Using identifiable locations on the rendered images, attempt was made to approximate the same location for measurement in both scans. These measurements are shown in Table 1, with 'Pre' being measurements taken from the first scan, and 'Post' being measurements taken following 6 months of healing post extraction and ridge preservation.

\begin{tabular}{lllll}
\hline \multirow{2}{*}{ Site } & \multicolumn{3}{l}{ Combination Allograft } & DFDBA \\
\cline { 2 - 5 } & $\begin{array}{l}\text { Pre (in } \\
\mathbf{m m})\end{array}$ & $\begin{array}{l}\text { Post (in } \\
\mathbf{m m})\end{array}$ & $\begin{array}{l}\text { Pre (in } \\
\mathbf{m m})\end{array}$ & $\begin{array}{l}\text { Post (in } \\
\mathbf{m m})\end{array}$ \\
\hline 1 & 7.8 & 6.8 & 6.1 & 6.9 \\
2 & 7.5 & 7.7 & 6.2 & 6.5 \\
3 & 8.7 & 8.6 & 6.5 & 5.6 \\
4 & 13.4 & 12.2 & 8.6 & 7.6 \\
5 & 7.5 & 8.2 & 8.1 & 6.5 \\
6 & 9.1 & 8.8 & 8.6 & 8.3 \\
7 & 8.7 & 7.2 & 8.3 & 7.4 \\
8 & 7.3 & 6.2 & 9.4 & 8.2 \\
9 & 8.1 & 6.9 & 6.2 & 5.4 \\
10 & 7.2 & 6 & 6.1 & 5.3 \\
Mean & 8.53 & 7.86 & 7.41 & 6.67 \\
Net Change & -0.67 & & -0.74 & \\
\hline
\end{tabular}

As is evident in Table 1, extraction followed by ridge preservation still led to some loss of alveolar ridge width as a general trend, although certain sites gained some width. With the test material, net loss in width was $0.67 \mathrm{~mm}$, and with the control group using demineralized freeze-dried bone allograft, net loss in width was $0.74 \mathrm{~mm}$ (Figure 1) (Figure 2).

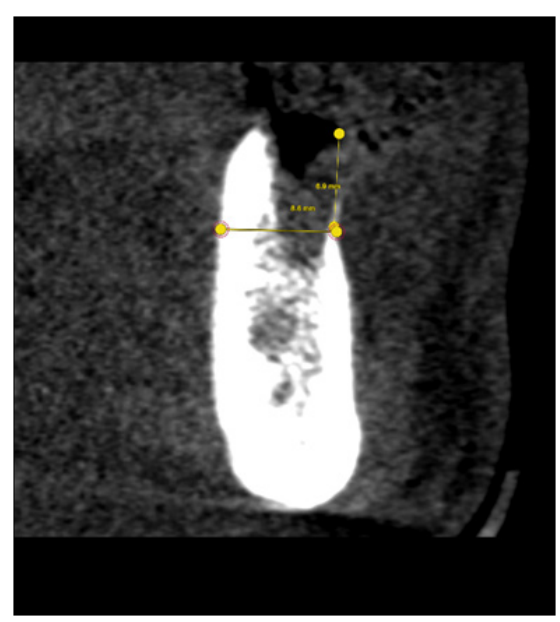

Figure I It shows cross-sectional images from a CBCT rendering immediately following extractions. Extraction outline can be seen clearly. This demonstrates how the measurements were made starting from the point where a thick, dense, radiolucent buccal plate was seen. Both these sites were grafted with DFDBA. 

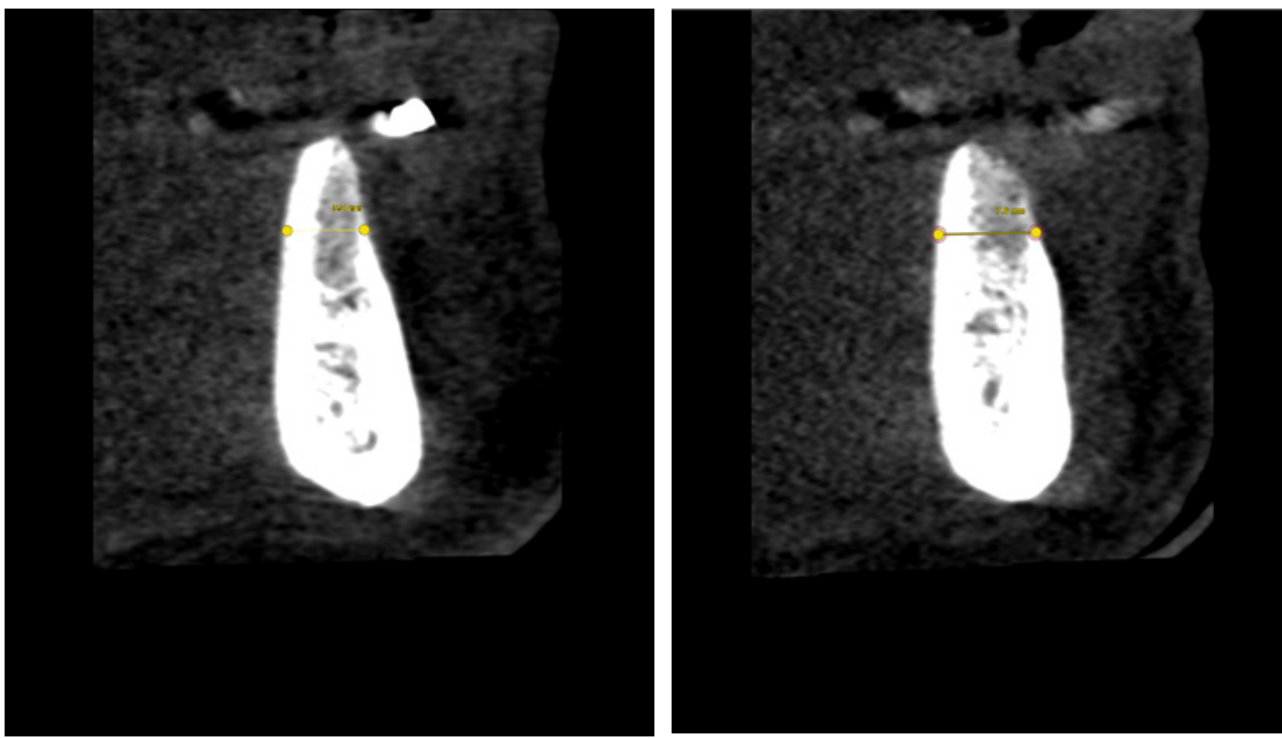

Figure 2 It shows cross-sectional images from a CBCT rending following 6months of healing for those same extraction sockets from Figure I. It can be seen that with DFDBA, graft integration is excellent.

\section{Discussion}

The present study compared alveolar ridge preservation following extraction using two different bone substitute materials. The control was demineralized freeze-dried bone allograft. Particular size for this graft material ranged from 0.25 to $1 \mathrm{~mm}$. The test sites received a fairly new combination allograft. This combination graft is a blend of $70 \%$ mineralized freeze-dried and $30 \%$ demineralized freeze-dried cortical bone allograft. The particular size ranged from 0.25 to $1 \mathrm{~mm}$.

During ridge preservation procedures, practitioners, with their ingenuity, often combine FDBA and DFDBA, hoping to obtain the positive aspects of each type of graft. This practice is not based on a specific clinical study, but rather, is based on there own clinical judgment. No other study has compared combination allograft to DFDBA in alveolar ridge preservation studies utilizing site-specific limited volume cone beam volumetric tomography scans. Based on the results, it can be noted that despite our best efforts to preserve the ridge, some dimensional change is bound to occur. This change was found as a loss of $0.67 \mathrm{~mm}$ for the test group and $0.74 \mathrm{~mm}$ for the control group. The fact that there was a dimensional loss in the alveolar ridge despite ridge preservation techniques coincides with existing literature. ${ }^{1-4}$ The amount of horizontal loss seen in this study seems to be less than what has been presented in other studies involving ridge preservation. ${ }^{4}$ This could be due to measurement errors in the software rendered images. It could also be due to the small sample size in this study. More accuracy could have been gained by fabricating a radiographic stent to be worn by the patient in both CBCT imaging sessions. This stent could then be scanned separately, and then combined with both scans of the patient at baseline and 6months following extraction and ridge preservation. Using this method, exactly identical positions could be selected based on the radiographic stent to make measurements. Despite not having such a stent, the measurement positions on the CBCT sectional images were fairly similar in both baseline and 6-month post-op scans. More accuracy could also have been gained by making clinical measurements immediately following extraction, and again at the time of implant placement. The change measured clinically could also have been compared to the changes measured via CBCT. This would have not only provided a second measure of alveolar ridge dimension change, it would have also served to measure the accuracy of CBCT measurements. Nonetheless, based on the results of this study, changes in alveolar ridge dimension are minimal when alveolar ridge preservation is done. Also, there are negligible differences in changes to alveolar ridge dimensions, when comparing DFDBA to combination allograft. Further, implant placement was possible without any additional augmentation in these sites that underwent alveolar ridge preservation.

A recent study from 2015 compared combination allograft to freeze-dried bone allograft (FDBA), and this group concluded that there are no significant differences between both groups when evaluating changes in ridge dimension. ${ }^{5}$ However, there was more vital bone in the combination allograft group compared to FDBA. FDBA also had significantly higher percentage of residual graft particles. ${ }^{5}$ This is clinically significant because it showed that the use of combination allograft led to more vital bone, at the same time, preserving similar alveolar ridge dimensions as FDBA. Another study compared FDBA to DFDBA in alveolar ridge preservation techniques. ${ }^{6}$ Again; the conclusion was that there were no significant differences in the two materials when evaluating changes in alveolar ridge width and height. However, similar to Borg's study, this group did find significantly higher percentage of vital bone in the DFDBA group compared to the FDBA group. ${ }^{5,6}$ The FDBA group also had significantly higher residual graft particles. Borg's study is favoring the use of combination allograft over FDBA; since the combination allograft group had more vital bone, and had similar alveolar ridge dimensional changes as FDBA. ${ }^{5}$ Wood's study is favoring DFDBA over FDBA for that same reasons. ${ }^{6}$ The present study is the first to evaluate combination allograft to DFDBA. However, these evaluation only included dimensional changes measured via $\mathrm{CBCT}$ and graft incorporation via $\mathrm{CBCT}$ and clinical examination, and did not include any histological evaluations. Future studies should include histological evidence to better support the use of one material over the other. ${ }^{7-14}$ 


\section{Conclusion}

It can be concluded that extraction followed by alveolar ridge preservation using either demineralized freeze-dried bone allograft or a 70:30 combination allograft resulted in minimal and clinically negligible changes in alveolar ridge dimensions. It can also be concluded that there were minimal and clinically negligible differences between the two grafting materials when measuring changes in alveolar ridge dimensions. CBCT scans showed excellent graft integration for both materials, and implants were placed in sites grafted with both materials without any need for additional augmentation. Larger sample size and histomorphometric analysis should be done in future studies to confirm these findings.

\section{Acknowledgements}

None.

\section{Conflict of interest}

The author declares no conflict of interest.

\section{References}

1. Horowitz R, et al. A Review on Alveolar Ridge Preservation Following Tooth Extraction. Journal of Evidence-Based Dental Practice. 2012;12:149-160.

2. Barone A, et al. Tissue changes of extraction sockets in humans: a comparison of spontaneous healing vs. ridge preservation with secondary soft tissue healing. Clinical Oral Implants Research. 2013;24:1231-1237.

3. Horvath A, et al. Alveolar ridge preservation. A systematic review. Clinical Oral Investigations. 2013;17:341-363.

4. Willenbacher M, et al. The Effects of Alveolar Ridge Preservation: A Meta-Analysis. Clinical Implant Dentistry and Related Research. 2016;18(6):1248-1268.
5. Borg TD, et al. Histologic healing following tooth extraction with ridge preservation using mineralized versus combined mineralizeddemineralized freeze-dried bone allograft: a randomized controlled clinical trial. Journal of Periodontology. 2015;86(3):348-355.

6. Wood RA, et al. Histologic comparison of healing after tooth extraction with ridge preservation using mineralized versus demineralized freezedried bone allograft. Journal of Periodontology. 2012;83(3):329-336.

7. Atwood DA. Reduction of residual ridges: A major oral disease entity. Journal of Prosthetic Dentistry. 1971;26(3):266-279.

8. Barone A, et al. Flap versus flapless procedure for ridge preservation in alveolar extraction sockets: a histological evaluation in a randomized clinical trial. Clinical Oral Implants Research. 2015;26:806-813.

9. Cosyn J, et al. Predictors of Alveolar Process Remodeling Following Ridge Preservation in High-Risk Patients. Clinical Implant Dentistry and Related Research. 2016;18(2):226-233.

10. Suttapreyasri S, et al. Influence of Platelet-Rich Fibrin on Alveolar Ridge Preservation. The Journal of Craniofacial Surgery. 2013;24(4):10881094.

11. Leblebicioglu B, et al. Determinants of alveolar ridge preservation differ by anatomic location. Journal of Clinical Periodontology. 2013;40:387395.

12. Lee A, et al. The Clinical Effectiveness of Alveolar Ridge Preservation in the Maxillary Anterior Esthetic Zone-A Retrospective Study. Journal of Esthetic and Restorative Dentistry. 2017;29(2):137-145.

13. Mardas N, et al. Does ridge preservation following tooth extraction improve implant treatment outcomes: a systematic review: Group 4: Therapeutic concepts \& methods. Clinical Oral Implants Research. 2015;26(11):180-201.

14. Atwood DA. Reduction of residual ridges: A major oral disease entity. Journal of Prosthetic Dentistry. 1971;26(3):266-279. 